\title{
Evaluation of cardiovascular drugs in post renal transplant patients
}

\section{Shouvik Choudhury ${ }^{1}$, Suparna Chatterjee ${ }^{2}$, Arpita Ray Chaudhury ${ }^{3}$}

${ }^{I}$ Pharmacology, post graduate trainee, IPGME\&R, Kolkata, India, ${ }^{2}$ Dept. of Pharmacology, IPGME\&R, Kolkata, India, ${ }^{3}$ Dept of Nephrology, IPGME\&R, Kolkata, India

\section{Background}

Renal transplant recipients remain at higher risk of developing cardiovascular (CV) diseases - Patient survival depends on optimum control of various risk factors for developing CV diseases along with proper graft functioning $\cdot$ Most common cause of mortality among these patients is also due to CV diseases . This observational study was undertaken to evaluate the prescribing pattern of drugs used for common cardiovascular diseases and also to evaluate their clinical effectiveness and safety profile ·

Methods

Patients who had undergone renal transplant from August 2014 to September 2016 in this public hospital were enrolled as per eligibility criteria. Each enrolled subject was followed up for a period of up to 12 months from the date of transplant. Demography, transplant details, cardiovascular drug usage, immunosuppressant intake profiles and clinical and laboratory profile were noted and analyzed.

Results

Total 56 patients were enrolled . The mean age of patients at transplant was $33.04 \pm 9.96$ years with male preponderance $(87 \cdot 5 \%) \cdot 25 \%$ patients required no antihypertensive at time of discharge and number of drug requirement gradually reduced - Analysis of number of drugs between pre-transplant time versus at discharge and 12 months post-transplant was statistically significant ( $\mathrm{p}$ value \&amplt0.0001) using Chi square test · Most common group of drug was CCB (namely Amlodipine) - Target BP (\&ample $130 / 80 \mathrm{~mm}$ of $\mathrm{Hg}$ ) was achieved in $62.5 \%$ patients at 1year post transplant period. $12.5 \%$ patients developed dyslipidemia and 25\% developed NODAT at 1 year post transplant - No mortality or incidence of MACE (major adverse cardiac event) occurred in this period. Hospitalization due to clinically significant medical events was noted in $15(26 \cdot 78 \%)$ subjects .

Conclusions

Prediction of modifiable risk factors, proper post transplant cardiovascular monitoring and adequate treatment can substantially reduce the incidence of CVD in post transplant patients - Incidence of hypertension and drug number gradually decreases. Few patients developed dyslipidemia and NODAT which may be attributed to the concomitant immunosuppressant use - Treatment protocol for each hospital should be framed and continuous long term prospective surveillance on drug usage pattern shall provide further insights · 